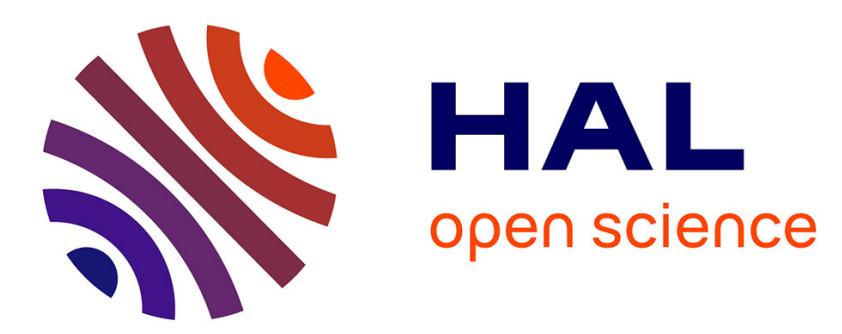

\title{
Modeling the effective elastic behavior of composites: a mixed Finite Element and homogenisation approach
}

\author{
Charles Toulemonde, Renaud Masson, Joumana El Gharib
}

\section{To cite this version:}

Charles Toulemonde, Renaud Masson, Joumana El Gharib. Modeling the effective elastic behavior of composites: a mixed Finite Element and homogenisation approach. Comptes Rendus Mécanique, 2008, 336 (3), pp.275 - 282. 10.1016/j.crme.2007.11.024 . hal-03427489

\section{HAL Id: hal-03427489 \\ https://hal.science/hal-03427489}

Submitted on 13 Dec 2021

HAL is a multi-disciplinary open access archive for the deposit and dissemination of scientific research documents, whether they are published or not. The documents may come from teaching and research institutions in France or abroad, or from public or private research centers.
L'archive ouverte pluridisciplinaire HAL, est destinée au dépôt et à la diffusion de documents scientifiques de niveau recherche, publiés ou non, émanant des établissements d'enseignement et de recherche français ou étrangers, des laboratoires publics ou privés. 


\title{
Modeling the effective elastic behavior of composites: a mixed Finite Element and homogenisation approach
}

\author{
Charles Toulemonde ${ }^{\mathrm{a}}$, Renaud Masson ${ }^{\mathrm{a}, *}$, Joumana El Gharib ${ }^{\mathrm{b}}$ \\ ${ }^{a}$ Materials and Mechanics of Components Department, EDF R\&D, avenue des Renardières, 77818 Moret-sur-Loing cedex, France \\ ${ }^{\mathrm{b}}$ Analysis in Mechanics and Acoustic Department, EDF R\&D, 1, avenue du Général-de-Gaulle, 92141 Clamart cedex, France
}

Received 14 September 2007; accepted after revision 27 November 2007

Available online 10 January 2008

Presented by André Zaoui

\begin{abstract}
To make possible the Finite Element computation of Representative Volume Elements (RVE) of composites with a complex microstructure, we first propose to mesh the volume independently of the considered microstructure. Homogeneous elastic properties are then given to the elements whose behavior is ill-defined (different phases in the same element) while a polarization is homogeneously applied to these so-called 'interphase elements' to control the corresponding error. New bounds are derived by optimizing the polarisation. When interphase elements are given extreme elastic properties these bounds yield satisfying results in both cases of a unit cell as well as a concrete RVE. To cite this article: $C$. Toulemonde et al., C. R. Mecanique 336 (2008).

๑) 2007 Académie des sciences. Published by Elsevier Masson SAS. All rights reserved.
\end{abstract}

\section{Résumé}

Modélisation du comportement élastique effectif des composites : approche mixte homogénéisation/éléments finis. Pour rendre possible les calculs (méthode des éléments finis) de Volumes Elémentaires Représentatifs (VER) de composites présentant une microstructure complexe, nous proposons de mailler indépendamment de la microstructure le volume considéré. Des propriétés élastiques homogènes sont ensuite affectées aux éléments dont le comportement est mal défini (éléments contenant des phases différentes), l'erreur commise étant contrôlée par l'application d'une polarisation, elle aussi homogène, dans ces éléments «interphases». De nouvelles bornes sont finalement exhibées en optimisant cette polarisation. Lorsque les éléments interphases possèdent des propriétés élastiques extrêmes, ces bornes conduisent à des encadrements probants (calculs de cellule puis VER de béton). Pour citer cet article :C. Toulemonde et al., C. R. Mecanique 336 (2008).

๑ 2007 Académie des sciences. Published by Elsevier Masson SAS. All rights reserved.

Keywords: Computational solid mechanics; Finite element; Homogenisation; Variational method; Sieve curves; Concrete; Microstructure; RVE

Mots-clés : Mécanique des solides numérique ; Eléments finis ; Homogénéisation ; Méthode variationnelle ; Fuseau granulaire ; Béton ;

Microstructure; VER

\footnotetext{
* Corresponding author.

E-mail addresses: charles.toulemonde@edf.fr (C. Toulemonde), renaud.masson@edf.fr(R. Masson), joumana.el-gharib@edf.fr (J. El Gharib).
} 


\section{Introduction}

This work deals with the problem of estimating the effective behavior of random heterogeneous media using Finite Element computations. Hereafter, a Representative Volume Elements (RVE) $V$ of a given heterogeneous material made of $n_{p}$ different homogeneous constituents is studied. Consider the boundary value problem for the volume $V$ on whose surface $\partial V$ an averaged strain $\overline{\boldsymbol{\varepsilon}}$ is homogeneously applied. The elastic moduli of each phase $(r)$ are denoted by the fourth order tensors $L^{r}\left(1 \leqslant r \leqslant n_{p}\right)$. The strain energy density function at any point $x \in V$ is defined by:

$$
w(x, \boldsymbol{\varepsilon})=\frac{1}{2} \boldsymbol{\varepsilon} \boldsymbol{L}(x) \boldsymbol{\varepsilon}
$$

with $\varepsilon$ the infinitesimal strain (second order) tensor. The effective property of the RVE is determined by its effective energy function [1]:

$$
\bar{W}(\overline{\boldsymbol{\varepsilon}})=\min _{\boldsymbol{\varepsilon} \in K} \frac{1}{|V|} \int_{V} w(x, \boldsymbol{\varepsilon}) \mathrm{d} x
$$

where $K$ denotes a set of kinematically admissible strain field consistent with the averaged strain $\bar{\varepsilon}$. This boundary value problem can be solved by Finite Element computations. However, computations of such random media needs a precise mesh of the microstructure. In practice, it may be intractable to mesh the RVE with respect to the complex microstructure of heterogeneous materials like concrete (inclusions are very numerous, the distance between inclusions may be very small ...). More generally, accurate simulations of realistic microstructures of random composites are CPU time consuming. These limitations can be overcome to some extent through the use of the new method proposed in the following.

\section{Principle of a mixed Finite Element and homogenisation approach}

\subsection{A homogeneous mesh of the RVE}

To avoid the meshing problem for such RVE and to make mechanical computation easier, a homogeneous mesh of the RVE volume $V$ has been adopted (the mesh can be free or regular). Then, any model of the microstructure under consideration is 'projected' on this homogeneous mesh (see, for instance, [2]). The elements of this mesh are divided as follows:

- elements that are exactly included in one of the $n_{p}$ phases (constitute the volume $V_{1}$ ),

- 'interphase elements' (constitute the volume $V_{2}$ ) that contain different phases (for instance both in matrix and inclusions for a two-phase composite).

This new boundary value problem is ill-posed: the elastic behavior of the volume $V_{2}$ is not clearly defined. If extreme properties are given to the volume $V_{2}$ (either the ones of the stiffer or softer phase), Finite Element computations will allow to derive bounds for the effective property (R. Hill's Strengthening Theorem [1], see also [3]). In the next section, we propose to define the elastic behavior of the volume $V_{2}$ in a different way to derive tighter bounds as well as estimates for the overall properties.

\subsection{Bounds and estimates of the effective properties}

Throughout this volume $V_{2}$, the energy function is approximated by:

$$
w(x, \boldsymbol{\varepsilon}) \approx \frac{1}{2} \boldsymbol{\varepsilon} \boldsymbol{L}^{0} \boldsymbol{\varepsilon}+\boldsymbol{p}^{0} \boldsymbol{\varepsilon}
$$


with uniform and symmetric fourth-order moduli tensor $\boldsymbol{L}^{0}$ as well as second-order tensor (of polarization) $\boldsymbol{p}^{0}$. The use of $\boldsymbol{L}^{0}$ and $\boldsymbol{p}^{0}$ is consistent with the work of Hashin and Shtrikman [4], but this approximation is restricted to the volume $V_{2}$ of interphase elements. Denoting by $c_{2}=\frac{\left|V_{2}\right|}{|V|}$, let $\xi$ denote:

$$
\xi=\frac{c_{2}}{\left|V_{2}\right|} \int_{V_{2}}\left(w(x, \boldsymbol{\varepsilon}(x))-\frac{1}{2} \boldsymbol{\varepsilon}(x) \boldsymbol{L}^{0} \boldsymbol{\varepsilon}(x)-\boldsymbol{p}^{0} \boldsymbol{\varepsilon}(x)\right) \mathrm{d} x=\frac{c_{2}}{\left|V_{2}\right|} \int_{V_{2}}\left(\frac{1}{2} \boldsymbol{\varepsilon}(x)\left(\boldsymbol{L}(x)-\boldsymbol{L}^{0}\right) \boldsymbol{\varepsilon}(x)-\boldsymbol{p}^{0} \boldsymbol{\varepsilon}(x)\right) \mathrm{d} x
$$

Assuming that $\boldsymbol{L}^{0}>\boldsymbol{L}^{+}=\max _{r}\left(\boldsymbol{L}^{r}\right)$ in the sense of the associated quadratic forms, the following inequality holds:

$$
\xi \leqslant \frac{c_{2}}{\left|V_{2}\right|} \int_{V_{2}} \max _{\boldsymbol{\varepsilon}}\left(\frac{1}{2} \boldsymbol{\varepsilon}\left(\boldsymbol{L}(x)-\boldsymbol{L}^{0}\right) \boldsymbol{\varepsilon}-\boldsymbol{p}^{0} \boldsymbol{\varepsilon}\right) \mathrm{d} x=\frac{1}{2} \boldsymbol{p}^{0}\left(\frac{c_{2}}{\left|V_{2}\right|} \int_{V_{2}}\left(\boldsymbol{L}^{0}-\boldsymbol{L}(x)\right)^{-1} \mathrm{~d} x\right) \boldsymbol{p}^{0}
$$

As a result, the effective energy function admits an upper bound, namely:

$$
\begin{aligned}
\bar{W}(\overline{\boldsymbol{\varepsilon}}) \leqslant & \min _{\boldsymbol{\varepsilon} \in K}\left(\frac{c_{1}}{\left|V_{1}\right|} \int_{V_{1}} w(x, \boldsymbol{\varepsilon}) \mathrm{d} x+\frac{c_{2}}{\left|V_{2}\right|} \int_{V_{2}}\left(\frac{1}{2} \boldsymbol{\varepsilon}(x) \boldsymbol{L}^{0} \boldsymbol{\varepsilon}(x)+\boldsymbol{p}^{0} \boldsymbol{\varepsilon}(x)\right) \mathrm{d} x\right) \\
& +\frac{1}{2} \boldsymbol{p}^{0}\left(\frac{c_{2}}{\left|V_{2}\right|} \int_{V_{2}}\left(\boldsymbol{L}^{0}-\boldsymbol{L}(x)\right)^{-1} \mathrm{~d} x\right) \boldsymbol{p}^{0}
\end{aligned}
$$

$\left(c_{1}=1-c_{2}\right.$ ), for any choice of the polarization $p^{0}$ (a nil polarization gives an upper bound already derived by the Strengthening Theorem [1]). The right term of the previous inequality can be optimized over the polarization $\boldsymbol{p}^{0}$ to give:

$$
\bar{W}(\overline{\boldsymbol{\varepsilon}}) \leqslant \min _{\boldsymbol{\varepsilon} \in K}\left(\frac{c_{1}}{\left|V_{1}\right|} \int_{V_{1}} w(x, \boldsymbol{\varepsilon}(x)) \mathrm{d} x+\frac{c_{2}}{\left|V_{2}\right|} \int_{V_{2}} w^{0}(x, \boldsymbol{\varepsilon}(x)) \mathrm{d} x\right)
$$

$\left(w^{0}(\boldsymbol{\varepsilon})=\frac{1}{2}\left(\boldsymbol{\varepsilon} \boldsymbol{L}^{0} \boldsymbol{\varepsilon}+\boldsymbol{p}^{0} \boldsymbol{\varepsilon}\right)\right)$ where the optimal polarization satisfies the relation:

$$
\int_{V_{2}} \boldsymbol{\varepsilon}(x) \mathrm{d} x+\left(\int_{V_{2}}\left(\boldsymbol{L}^{0}-\boldsymbol{L}(x)\right)^{-1} \mathrm{~d} x\right) \boldsymbol{p}^{\mathbf{0}}=0
$$

The following comments can be made:

- The relation (6) is obtained by derivating the relation (5) with respect to the polarization and making use of the fact that $\int_{V} \frac{\partial}{\partial \boldsymbol{\varepsilon}}(.) \frac{\partial \boldsymbol{\varepsilon}}{\partial \boldsymbol{p}^{\mathbf{0}}} \mathrm{d} x$ vanishes (stationarity with respect to the strain field).

- The minimization in (5) denotes the effective energy function of an auxiliary 'thermo-elastic' problem where the polarization is uniform in the volume $V_{2}$. This new boundary value problem is well-posed and can be solved by Finite Element calculations.

- Combining the latest expression (6) with the average of the stress strain relation (3) over the volume $V_{2}$ yields:

$$
\int_{V_{2}} \boldsymbol{\sigma}(x) \mathrm{d} x=\hat{\boldsymbol{L}}^{0} \int_{V_{2}} \boldsymbol{\varepsilon}(x) \mathrm{d} x \quad \text { with } \hat{\boldsymbol{L}}^{0}=\boldsymbol{L}^{0}+\left(\frac{1}{\left|V_{2}\right|} \int_{V_{2}}\left(\boldsymbol{L}(x)-\boldsymbol{L}^{0}\right)^{-1} \mathrm{~d} x\right)^{-1}
$$

where $\hat{\boldsymbol{L}}^{0}$ is called hereafter the effective modulus in the volume $V_{2}$.

- Assuming that $\boldsymbol{L}^{0}<\boldsymbol{L}^{-}=\min _{r} \boldsymbol{L}^{r}$ changes the max to a min and the sign of inequality in relation (4), this leads to a lower bound of the effective energy function.

- If the condition $\boldsymbol{L}^{-} \leqslant \boldsymbol{L}^{0} \leqslant \boldsymbol{L}^{+}$is satisfied, right term of (5) should also provide variational estimates for the effective strain energy function. This last item will be examined in future works.

It now remains to derive the explicit expression of the optimal polarization related to the choice of a given elastic modulus $\boldsymbol{L}^{0}$ in the volume $V_{2}$. Let $\boldsymbol{\varepsilon}^{H(0)}$ be the strain field solution consistent with the averaged strain $\overline{\boldsymbol{\varepsilon}}$ applied on $V$ (and a nil polarization in $\left.V_{2}\right)$. The polarization can be decomposed on the orthonormal basis $\boldsymbol{e}_{I}(1 \leqslant I \leqslant N)$ as 
$\boldsymbol{p}^{0}=\sum_{I=1}^{I=N}\left(\boldsymbol{p}^{0} \boldsymbol{e}_{I}\right) \boldsymbol{e}_{I}$ where the number $N$ depends on the symmetry of the RVE $(N \leqslant 6)$. If $\boldsymbol{\varepsilon}_{I}^{r(0)}$ denotes the strain residual field experienced by the same volume $\left(\boldsymbol{L}(x)=\boldsymbol{L}^{0}\right.$ in $\left.V_{2}\right)$ when the RVE is free (strain free) at its boundary $\partial V$ and is submitted to the polarization $C^{0} \boldsymbol{e}_{I}$ in the volume $V_{2}\left(C^{0}\right.$ denotes an arbitrary constant), the superposition Theorem ensures that:

$$
\boldsymbol{\varepsilon}=\boldsymbol{\varepsilon}^{H(0)}+\frac{1}{C^{0}} \sum_{I=1}^{I=N}\left(\boldsymbol{p}^{0} \boldsymbol{e}_{I}\right) \boldsymbol{\varepsilon}_{I}^{r(0)}
$$

$\boldsymbol{\varepsilon}$ is the strain field in $V$ consistent both with the averaged strain $\overline{\boldsymbol{\varepsilon}}$ applied on its boundary and with the polarization $\boldsymbol{p}^{0}$ uniformly applied in $V_{2}$. Elementary fields $\varepsilon^{H(0)}$ and $\varepsilon_{I}^{r(0)}$ can be derived by Finite Element calculations. Reporting expression (8) into Eq. (6) leads to:

$$
\boldsymbol{p}^{0}=-\left(\int_{V_{2}}\left(\frac{1}{C^{0}} \sum_{I=1}^{I=N} \boldsymbol{\varepsilon}_{I}^{r(0)}(x) \otimes \boldsymbol{e}_{I}+\left(\boldsymbol{L}^{0}-\boldsymbol{L}(x)\right)^{-1}\right) \mathrm{d} x\right)^{-1} \int_{V_{2}} \boldsymbol{\varepsilon}^{H(0)}(x) \mathrm{d} x
$$

Substituting $\boldsymbol{L}^{0}$ by $\boldsymbol{L}^{0-} \leqslant \boldsymbol{L}^{-}$or $\boldsymbol{L}^{0+} \geqslant \boldsymbol{L}^{+}$in the former expression yields the polarization associated to lower and upper bounds respectively. The computations of these two optimal polarizations require Finite Element calculations of the strain fields $\left(\boldsymbol{\varepsilon}^{H(-)}, \boldsymbol{\varepsilon}_{1}^{r(-)}, \ldots, \boldsymbol{\varepsilon}_{N}^{r(-)}\right)$ and $\left(\boldsymbol{\varepsilon}^{H(+)}, \boldsymbol{\varepsilon}_{1}^{r(+)}, \ldots, \boldsymbol{\varepsilon}_{N}^{r(+)}\right)$, respectively.

\subsection{Optimal homogeneous elastic moduli tensor in $V_{2}$}

When $\boldsymbol{L}^{0}$ tends to $\boldsymbol{L}^{+}$, the fields $\left(\boldsymbol{\varepsilon}^{H(0)}, \boldsymbol{\varepsilon}_{1}^{r(0)}, \ldots, \boldsymbol{\varepsilon}_{N}^{r(0)}\right)$ remain bounded throughout $V_{2}$ while the difference $\left(\boldsymbol{L}^{0}-\boldsymbol{L}(x)\right)$ tends to zero at point $x$ where $\boldsymbol{L}(x)=\boldsymbol{L}^{+}$. Let us note $V_{2}^{+}$this part of $V_{2}$, a first order expansion of the optimal polarization (relation (9)) gives:

$$
\boldsymbol{p}^{0} \approx \frac{1}{\left|V_{2}^{+}\right|}\left(\boldsymbol{L}^{+}-\boldsymbol{L}^{0}\right) \int_{V_{2}} \boldsymbol{\varepsilon}^{H(0)}(x) \mathrm{d} x=\boldsymbol{O}\left(\boldsymbol{L}^{+}-\boldsymbol{L}^{0}\right)
$$

then the bounds are reduced to the ones derived by the Strengthening Theorem when $\boldsymbol{L}^{0}=\boldsymbol{L}^{+}$( or $\boldsymbol{L}^{0}=\boldsymbol{L}^{-}$). Conversely, when $\boldsymbol{L}^{0}$ tends to infinity, the optimal polarization remains bounded while the effective modulus $\hat{\boldsymbol{L}}^{0}$ in $V_{2}$ (relation (7)) is reduced to Voigt's upper bound on this specific volume ( $\boldsymbol{I}$ is the unit fourth order tensor):

$$
\hat{\boldsymbol{L}}^{0} \approx \boldsymbol{L}^{0}\left(\boldsymbol{I}-\left(\boldsymbol{I}+\left(\frac{1}{\left|V_{2}\right|} \int_{V_{2}} \boldsymbol{L}(x) \mathrm{d} x\right) \boldsymbol{L}^{(0)^{-1}}\right)^{-1}\right) \approx \frac{1}{\left|V_{2}\right|} \int_{V_{2}} \boldsymbol{L}(x) \mathrm{d} x
$$

For any value of $\boldsymbol{L}^{0}$ in the interval $\left[\boldsymbol{L}^{+} ;+\infty\left[, \frac{\mathrm{d} \hat{\boldsymbol{L}}^{0}}{\mathrm{~d} \boldsymbol{L}^{0}}=\boldsymbol{I}-\boldsymbol{H}^{0} \leqslant \mathbf{0}\right.\right.$ with the fourth order tensor $\boldsymbol{H}^{0}$ defined as:

$$
\begin{aligned}
\boldsymbol{H}^{0} & =\left(\int_{V_{2}}\left(\boldsymbol{L}^{0}-\boldsymbol{L}(x)\right)^{-2} \mathrm{~d} x\right)\left(\int_{V_{2}}\left(\boldsymbol{L}^{0}-\boldsymbol{L}(x)\right)^{-1} \mathrm{~d} x\right)^{-2} \\
& \geqslant\left(\int_{V_{2}}\left(\boldsymbol{L}^{0}-\boldsymbol{L}(x)\right)^{-1} \mathrm{~d} x\right)^{2}\left(\int_{V_{2}}\left(\boldsymbol{L}^{0}-\boldsymbol{L}(x)\right)^{-1} \mathrm{~d} x\right)^{-2}=\boldsymbol{I}
\end{aligned}
$$

(with the notation $\boldsymbol{A}^{p} \boldsymbol{A}^{q}=\boldsymbol{A}^{p+q}$ for any fourth order tensor $\boldsymbol{A}$ and integers $(p, q)$ ). It follows that the effective modulus decreases from the upper elastic modulus $\boldsymbol{L}^{+}$to the Voigt bound over the volume $V_{2}\left(\frac{1}{\left|V_{2}\right|} \int_{V_{2}} \boldsymbol{L}(x) \mathrm{d} x\right)$ when $\boldsymbol{L}^{0}$ is increased from $\boldsymbol{L}^{+}$to $+\infty$. The sharper upper bound would probably be obtained for the lowest effective modulus in $V_{2}$ (but this qualitative argument is not a proof). As a result the choice of a (quasi)-rigid modulus in $V_{2}\left(\boldsymbol{L}^{0} \gg \boldsymbol{L}^{+}\right)$will be adopted as the optimal upper bound in next simulations. Conversely, the choice of a weak modulus in $V_{2}\left(\boldsymbol{L}^{0} \ll \boldsymbol{L}^{-}\right)$should provide the optimal lower bound associated to an effective modulus on $V_{2}$ equal to the Reuss lower bound on this sub-volume. These new bounds are called hereafter 'HST' as 'Hashin-Shtrikman 
Type' for obvious reasons and should be tighter than those derived by R. Hill previously. Next section aims at studying quantitatively this question.

Finally, it is noted that former results have been derived for the particular boundary value problem (2) related to homogeneous (strain) boundary conditions. If now, we consider the general boundary value problem where external loading (mixed boundary conditions and body forces) are applied to volume $V$, the strain part of the potential energy can be bounded (or estimated) with the help of similar arguments. For homogeneous (stress) boundary conditions, the effective complementary energy can be bounded in exactly the same fashion.

\section{Results}

\subsection{Unit cell calculations}

Here, we apply the proposed methodology to a volume $V$ whose effective response can be determined exactly. This reference case consists of a cubic volume $V$. A spherical inclusion is located at the center of this unit cell and embedded in a homogeneous matrix. Both matrix and inclusion are isotropic and incompressible with shear moduli $\mu^{m}$ and $\mu^{i}$, respectively. The inclusion volume fraction $f$ is equal to $20 \%$. The volume $V$ is modeled by free meshes ranging from 3000 to 335000 elements (Fig. 1). To provide reference results, an exact mesh of this unit cell has also been generated. The corresponding Finite Element results are called 'exact results' hereafter.

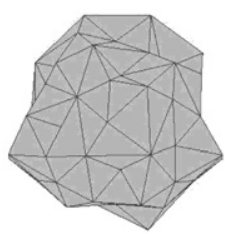

(a)

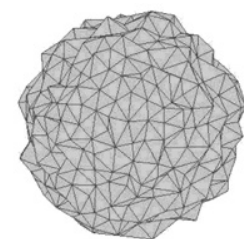

(b)

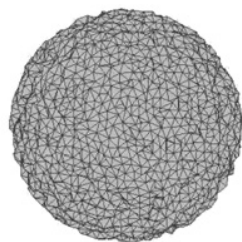

(c)

Fig. 1. Inclusion elements for three different sizes of meshing: (a) 3000 elements; (b) 32000 elements; (c) 335000 elements.

Fig. 1. Eléments de l'inclusion pour trois tailles de maille différentes : (a) 3000 éléments ; (b) 32000 éléments ; (c) 335000 éléments.

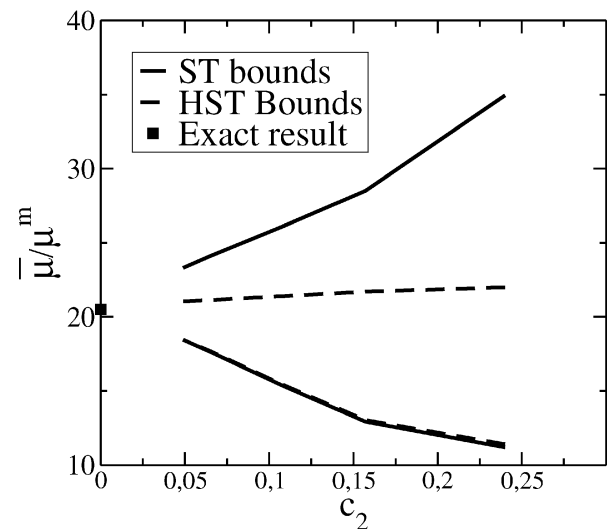

(a)

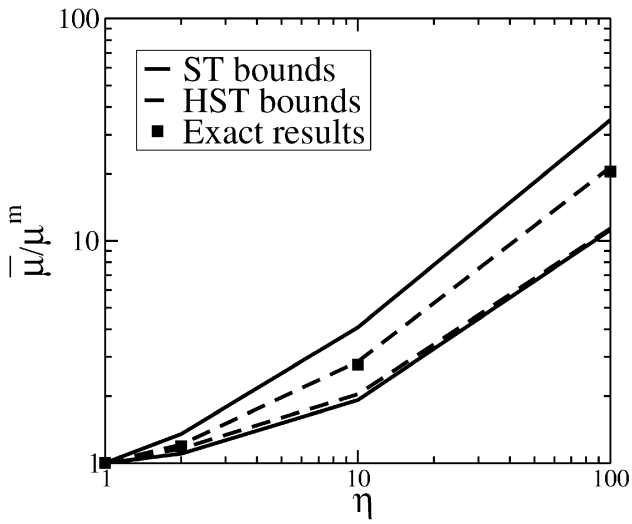

(b)

Fig. 2. (a) Overall modulus of the unit cell as a function of the volume fraction of interphase elements (contrast $\eta=100$ ): new bounds (HST) are compared to Hill's (ST) ones. The exact result is also reported (the filled square at $c_{2}=0$ ); (b) Overall modulus of the unit cell (crude free mesh) as a function of the contrast (logarithmic scales): new bounds (HST) are compared to Hill's ones (ST) and to exact results (the filled squares).

Fig. 2. (a) Evolution du module effectif de la cellule unitaire en fonction de la fraction volumique d'éléments d'interphase (contraste égal à 100) : comparaison des nouvelles bornes (HST) à celles de Hill (ST); le carré plein à $c_{2}=0$ correspondant au résultat exact. (b) Evolution du module effectif en fonction du contraste (échelle logarithmique) dans le cas du maillage le plus grossier : comparaison des nouvelles bornes (HST) à celles de Hill (ST) ainsi qu'aux résultats exacts (carrés pleins). 


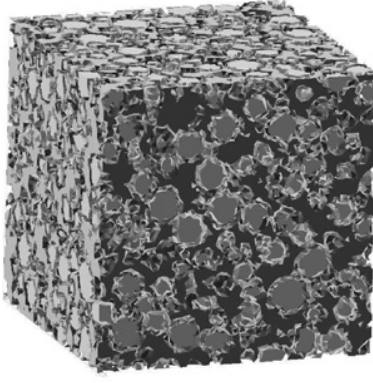

(a)

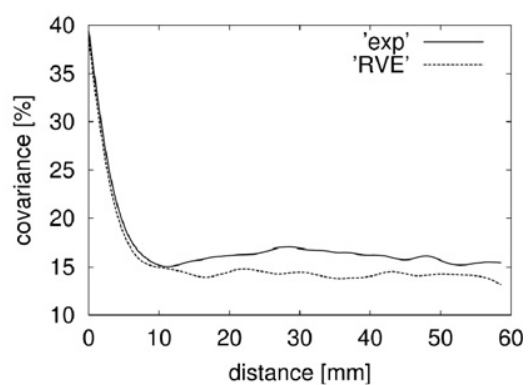

(b)

Fig. 3. (a) Pure inclusion and pure matrix elements of a concrete RVE meshing (one million elements); (b) Comparison between experimental and numerical covariograms.

Fig. 3. (a) Volume $V_{1}$ d'un maillage de VER de béton (un million d'éléments); (b) Comparaison du covariogramme expérimental à celui du VER simulé.

As a result of constituent properties and the spherical shape of the inclusion, the overall behavior of this unit cell is incompressible and displays a cubic symmetry. However, due to the low volume fraction of the inclusion, the effective responses computed in next simulations deviate slightly from an isotropic behavior. As a consequence, the (incompressible) effective behavior of this unit cell is entirely defined by its overall shear modulus $\bar{\mu}$. Notice that the overall modulus depends on the boundary conditions since this unit cell is not actually a true RVE as it should be for periodic homogenisation. Hereafter a homogeneous shear stress is applied on the unit cell's boundary. On Fig. 2(a) the evolutions of the overall shear modulus are reported as a function of the volume fraction of interphase elements $c_{2}=\frac{V_{2}}{V}$ (for a given contrast $\eta=100$ ). As expected, the bounds derived from R. Hill's Strengthening Theorem (ST) as well as the new (HST) bounds tend to the exact result when the volume fraction of interphase elements vanishes. For such a high contrast value the new lower bound is very close to the one derived by the Strengthening Theorem. By contrast the new upper bound displays small variations with this volume fraction and yields results close to the exact one. The new upper bound gives satisfying results even when derived from the crude free mesh (3000 elements, see Fig. 1(a)). In this case the effect of the contrast $\eta=\frac{\mu^{i}}{\mu^{m}}$ on Hill (ST) as well as new (HST) bounds for the overall shear modulus were also investigated on Fig. 2(b). As expected, bounds deviate with respect to an increasing contrast while the new lower bound tends to the one derived by the Strengthening Theorem when the contrast increases. In addition, the new upper bound improves significantly the one derived by the Strengthening Theorem and yields results close to the exact ones.

\subsection{RVE calculations on a concrete microstructure}

\subsubsection{Microstructure generation and meshing}

To generate the concrete RVE, the sieving curve of the sand used to manufacture a real concrete is adopted. For practical reasons, and also because of the existence of a kind of scale break we only consider inclusions with diameter bigger than $6 \mathrm{~mm}$. As a consequence the matrix of our concrete RVE is relevant of a mortar. The mortar is constituted of a cement matrix reinforced by sand inclusions, its volume fraction is equal to 50\%. The elastic properties of the mortar could result from the homogenisation of a two phase composite or from experiment. In the present case an homogenisation method has been used [5].

A random set of inclusions is first generated. Like other authors we use a Monte Carlo or Poisson type method and fuller curve to generate this set of inclusions (see [6]). However, the difference regarding the morphology is that the process allows the inclusions to intersect the RVE boundaries. We also add a periodicity constraint to the morphology generation process. These additional features should lead to more realistic virtual microstructures. Here, the volume fraction of inclusions is $f=40 \%$, which implies a number of about 2000 inclusions in a $150 \mathrm{~mm}$ large concrete RVE. The mesh of the RVE is homogeneous and free, it is composed of tetrahedra: Fig. 3(a) gives a picture of a one million element meshing. The experimental and numerical co-variograms fit together (Fig. 3(b)). Their comparison gives a first estimate of the RVE morphology accuracy. Unlike [7] or other authors, our meshing method generates interphase 
Table 1

Overall shear modulus of the concrete RVE as a function of the volume fraction of interphase elements $c_{2}$ : new bounds (HST) are compared to Hill's (ST) ones

Tableau 1

Evolution du module effectif du VER de béton en fonction de la fraction volumique $c_{2}$ d'éléments d'interphase : comparaison des nouvelles bornes (HST) à celles de Hill (ST)

\begin{tabular}{lllll}
\hline$c_{2}$ & 0.46 & 0.49 & 0.62 & 0.71 \\
\hline $\bar{\mu} / \mu^{m}$ (ST lower bound) & 1.23 & 1.22 & 1.16 & 1.11 \\
$\bar{\mu} / \mu^{m}$ (HST lower bound) & 1.37 & 1.36 & 1.35 & 1.34 \\
$\bar{\mu} / \mu^{m}$ (HST upper bound) & 1.67 & 1.68 & 1.7 & 1.33 \\
$\bar{\mu} / \mu^{m}$ (ST upper bound) & 1.98 & 2.02 & 2.2 & 2.72 \\
\hline
\end{tabular}

elements whose elastic properties are ill-defined. The choice of the optimal properties for these elements is the subject of the theoretical developments explained above.

At this stage it is worth emphasizing that the proposed method makes possible a computation of a concrete RVE with 2000 inclusions and only 200000 tetrahedra elements while classical meshing methods require more than 700000 elements to represent less than 200 inclusions and with less ergodicity [8]. Alternatively, the application of the aligned meshing approach to the considered RVE will require to reach mesh sizes consistent with the lowest distance between inclusions (about five hundred less than the RVE overall size!). From a practical point of view (mesh generation, computation times and memory allocation), this problem may be intractable. The counterpart of the proposed method's efficiency is the volume $V_{2}$ of interphase elements which turns out to be high for such a microstructure (as a result of the high volume fraction of inclusions): $c_{2}$ ranges from $c_{2}=0.74$ (234000 elements) to $c_{2}=0.46$ (1370000 elements). In the next subsection, we study if the choice of optimal properties in $V_{2}$ (see Section 2.3) allows us to yield accurate predictions of the effective properties of the concrete RVE in spite of $c_{2}$ high values.

\subsubsection{Computation of the effective elastic properties}

The stiffness ratio between the matrix (i.e. mortar) and the inclusions (i.e. the aggregates) is rather small: the Young modulus of the inclusions $(61.4 \mathrm{GPa})$ is 2.8 times higher that one of the matrix $(22.0 \mathrm{GPa})$. The Poisson ratios of the matrix and the inclusions are respectively 0.24 and 0.28 . To compute effective overall elastic properties, the concrete RVE is submitted to an uni-axial compressive load. Additional calculations have shown that the effective properties computed in the sequel do not depend on the loading conditions (e.g. the direction of compression, the boundary conditions, ...).

In Table 1 the evolutions of the overall shear modulus as a function of $c_{2}$ are reported (similar results on the overall bulk modulus have been obtained). These results are consistent with those derived previously on the unit cell (see Section 3.1). In particular, the new (HST) bounds improve significantly the ones derived by the Strengthening Theorem, especially for large values of $c_{2}$ : for the cruder mesh, the uncertainty on the effective shear modulus value decreases from approximately $\pm 36 \%$ (ST bounds) to $\pm 13 \%$ (the new HST bounds).

\section{Conclusion}

In this Note a new method is proposed in order to overcome limitations of classical RVE simulations for complex heterogeneous microstructures. As a first idea the RVE is meshed independently of the microstructure to facilitate the meshing and to make the computation possible with a rather small number of elements. The second idea (i.e. the use of optimal properties (polarization and elastic moduli) in the interphase elements) provides bounds as well as estimates for the effective elastic properties. These bounds allow to control the error related to a given mesh precision. The two ideas make both efficient and rather accurate the computation of RVE with very complex microstructure. First applications on two-phases heterogeneous volumes (unit cell and model of concrete microstructures) yield encouraging results: the new bounds improve significantly former ones derived with the Strengthening Theorem [1]. Further applications and developments will be dedicated to the steady creep behavior of concretes. Next theoretical works should concern the derivation of estimates. 


\section{Acknowledgements}

J.M. Ricaud (CEA-DEN) and J.M. Proix (EDF-R\&D) are gratefully acknowledged for their valuable help concerning the FE computations. We are also grateful to Prof. A. Zaoui and Y. Le-Pape (EDF-R\&D) for providing references to the Strengthening Theorem and numerical computations on concrete, respectively.

\section{References}

[1] R. Hill, Elastic properties of reinforced solids: Some theoretical principles, J. Mech. Phys. Solids 11 (1963) 357-372.

[2] T.I. Zohdi, P. Wriggers, Aspects of the computational testing of the mechanical properties of microheterogeneous material samples, Int. J. Numer. Meth. Eng. 50 (2001) 2573-2599.

[3] C. Huet, P. Navi, P.E. Roelfstra, A homogenization technique based on Hill's modification theorem, in: G.A. Maugin (Ed.), Continuum Models and Discrete Systems, vol. 2, Longman, Harlow, 1991, pp. 135-143.

[4] Z. Hashin, S. Shtrikman, A variational approach to the theory of the elastic behaviour of multiphase materials, J. Mech. Phys. Solids 11 (1963) $127-140$.

[5] Y. Le Pape, What can be learnt from the use of homogenization techniques to derive the mechanical properties of concrete in an industrial context?, in: G.Z. Voyiadis, R. Dorgan (Eds.), Proceedings of the Joint ASME/ASCE/SES Conference on Mechanics and Materials, Baton Rouge, Louisiana, USA, 2005.

[6] Z.M. Wang, A.K.H. Kwan, H.C. Chan, Mesoscopic study of concrete I: generation of random aggregate structure and finite element mesh, Comput. Struct. 70 (5) (1999) 533-544.

[7] E. Schlangen, J.G.M. van Mier, Simple lattice model for numerical simulation of fracture of concrete materials and structures, Mater Struct. 25 (153) (1992) 534-542.

[8] P. Wriggers, S.O. Moftah, Mesoscale models for concrete: Homogenisation and damage behaviour, Finite Element Anal. Design 42 (2006) 623-636 